\title{
A new benchmark in biodiversity monitoring
}

\author{
Book Title: \\ Biodiversity in Southern \\ Africa. Volume 1-3 \\ ISBN: \\ 978-99916-57-30-1 \\ Publisher: \\ Klaus Hess Publishers: \\ Göttingen \& Windhoek; \\ 2010, R600* \\ *Book price at time of review \\ -

\section{Review Title:} \\ A new benchmark in \\ biodiversity monitoring

\section{Reviewer:} \\ Robert J. Scholes ${ }^{1}$

\section{Affiliation:} \\ ${ }^{1}$ CSIR Natural Resources and \\ Environment, Pretoria, \\ South Africa \\ Email: \\ bscholes@CSIR.co.za \\ Postal address: \\ PO Box 395, Pretoria 0001, \\ South Africa
}

How to cite this book review: Scholes, R.J., 2012, 'A new benchmark in biodiversity monitoring', Koedoe 54(1), Art. \#1109, 2 pages. http:// dx.doi.org/10.4102/koedoe. v54i1.1109

(C) 2012. The Authors. Licensee: OpenJournals Publishing. This work is licensed under the Creative Commons Attribution License.

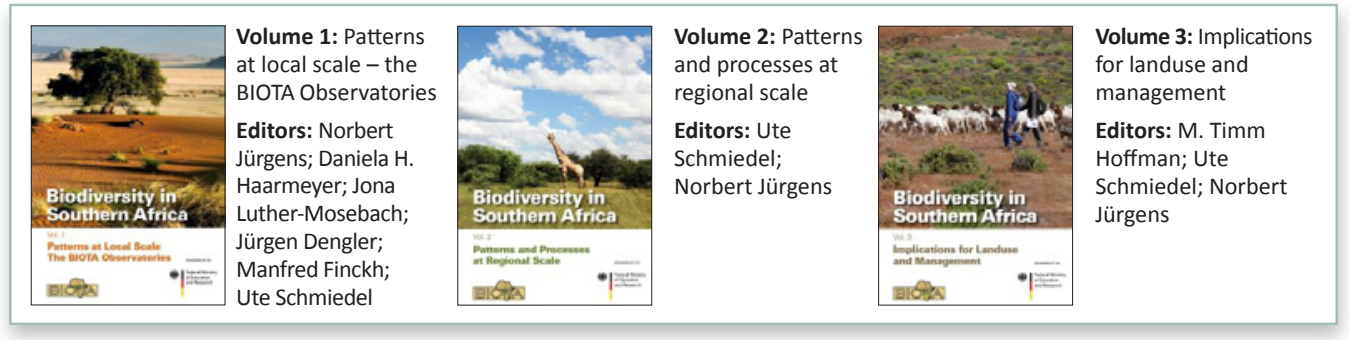

The BIOTA project began in South Africa and Namibia in the year 2000. It recently published a threevolume synthesis of its first decade of work. Unusually, 'BIOTA' is not an acronym for anything - it is the name of a major German-funded study into biodiversity patterns, initially focusing on the west side of southern Africa. BIOTA now has transects in North, West and East Africa as well.

BIOTA (http:/ / www.biota-africa.org) has been a pioneer in the field of 'biodiversity observatories' - in other words, locations where the biodiversity in all its forms and aspects is monitored over large scales and long periods of time. Conceptually, the BIOTA approach represents a large step up from the simple checklists taken in small plots that characterised the field previously. In a rough outline, the BIOTA network consists of $1 \mathrm{~km} \times 1 \mathrm{~km}$ squares, distributed strategically along subcontinental-scale environmental gradients. In the case of southern Africa, the network consists of 37 such squares; stretching from the high winter rainfall of the Cape Peninsula, through the drylands of Namaqualand and the Namib, to the high summer rainfall of northern Namibia. In some cases, the squares are paired at a location, representing contrasting land uses. The sampling design within the $1 \mathrm{~km}$ squares is elaborate. Each square is divided into 100 plots of 1 ha; nested within the plots are quadrats of $50 \mathrm{~m} \times 20 \mathrm{~m}$ and $10 \mathrm{~m} \times 10 \mathrm{~m}$ for vegetation sampling. Other parts of the plot are reserved for activities that cause disturbances (such as soil pits or trapping of small animals) or areas that must be protected from disturbance, such as the mapping of soil crusts. The plots are pre-classified using vegetation and geomorphological information from aerial photos or high-resolution remote sensing. A stratified-random sample of 20 plots is taken, with weighting to ensure that rare habitat types are also featured. Each square has an automatic weather station.

The detail of the information gathered has a mesmerising thoroughness. No stone is left unturned and no taxonomic group is too obscure to attract attention. Pedodiversity, biological soil crusts, lichens, vascular plants, millipedes, termites, bugs, beetles, butterflies, moths and vertebrates all have their own sample protocols. Even humans and their use of the land come under the microscope. It is perhaps not surprising that three hefty volumes, in colour, were needed to summarise this wealth of information. The real long-term value of this project probably esides in the primary observations themselves, and how they change over time. The original data is in the public domain, which is conveniently included as a CD at the back of volume 3 .

What emerges from this prodigious and costly effort? Volume 1 (801 pages) describes the methods and observed biodiversity patterns, site by site, within broad ecosystem clusters such as 'Woodland Savanna', 'Thornbush Savanna', 'Nama Karoo', 'Namib Desert', 'Succulent Karoo' and 'Fynbos'. It is beautifully laid out and illustrated, but the sheer volume of data is likely to overwhelm all but the most dedicated reader. Volume 2 is a more approachable 348 pages. It extracts the patterns and processes at regional scale, in the form of eight chapters, each with multi-authored subsections, covering topics ranging from land use history, through biogeography, contemporary human impacts, the use of experiments and models and capacity building. I suspect most readers will dip into the papers that most attract them, rather than reading cover-to-cover. Some interesting (but not completely unanticipated) patterns emerge. For instance, plant species richness - at all scales from $10 \mathrm{~m} \times 10 \mathrm{~m}$ to $1000 \mathrm{~m} \times 1000 \mathrm{~m}$ - declines with declining (summer) rainfall, is lowest in the desert and rises steeply with increasing winter rainfall to a maximum in the moist fynbos, at about double the score recorded in the wettest savannas. This pattern is much less obvious if the richness of 
plant families is considered. Deserts have substantially less than half the family richness of the other biomes, which differ little between them. About a third of the variation in plant species richness is accounted for by soil-type diversity. Moth diversity followed a similar relationship to precipitation as plants did, and reptile diversity increased with increasing grass cover.

Even a decade-long study is too short to say much about changes over time. There was a small increase in plant species richness over this period - so consistently across sites that I agree with the authors that it most probably reflects the growing observational skills of the botanists, rather than a fundamental change in the landscape. Interestingly, in this study land use intensity has no consistent impact on species richness.

Volume 3, a comparatively slim 226 pages in length, covers management implications. It emphasises the interdisciplinary nature of the BIOTA programme and includes modelled results from climate change studies as well as discussions of land use systems; in both cases only loosely linked to the BIOTA observatories and their data. I was slightly disappointed to find little that was really new or could only have been derived from a BIOTA-style system. It is perhaps unreasonable to expect novel insights from a young programme; nevertheless the pressure was obviously there to demonstrate how all that effort helped to guide decisionmaking, so something had to be said.

The BIOTA team have set an ambitious standard for biodiversity monitoring. The German research authorities continue to support the programme in its second decade, but have already signalled a desire that the within-region countries need to take over the long-term funding. Herein lies the rub: despite paying a laudable amount of attention to building local partnerships and capacity, it is not clear that this is the system that South Africa and Namibia (or the various other countries in Africa) would have built for their own purposes, and can afford to sustain. Will it survive to fulfil its long-term promise, or is this a luxury German vehicle in an environment where a bakkie would be more appropriate?

These volumes deserve a place in many institutional libraries in the region, especially those that deal with the ecosystems of the western part of the subcontinent. 